\title{
Neuartige Schmiedelinie für Magnesiumbauteile
}

Weisensee Warmpressteile errichtet eine neuartige Schmiedelinie. Installiert wird sie im Rahmen eines durch das Umweltinnovationsprogramm des Bundesumweltministeriums geförderten Pilotprojekts und soll die energetisch hocheffiziente Massenproduktion von Bauteilen aus Magnesiumlegierungen ermöglichen. Mithilfe des neuen Verfahrens könnten beispielsweise aus Magnesiumknetlegierungen Strukturbauteile für Kraftfahrzeuge hergestellt werden.

Die Schmiedelinie des im hessischen Eichenzell ansässigen Unternehmens ist auf

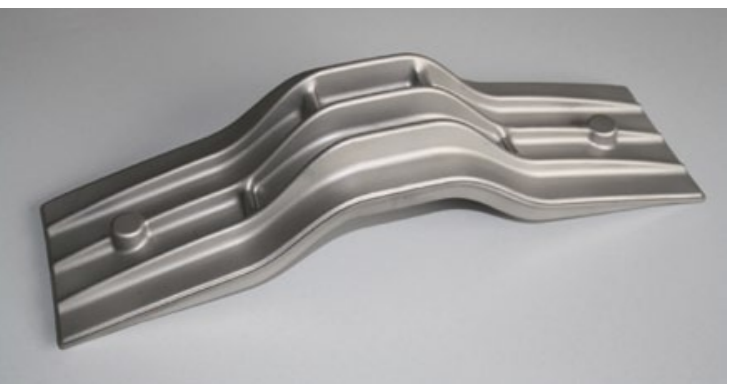

die verfahrens- und werkstofftechnischen Bedürfnisse von Magnesium abgestimmt, heißt es. Das Verfahren ermögliche erstmals, komplexe Bauteile mit hohem Integrationspotenzial und extrem belastungsangepasster Materialverteilung herzustellen. Für die Fertigung der Bauteile komme ein kontinuierlich arbeitender Durchlaufofen mit Porenbrennertechnologie zum Einsatz. Bislang seien Umluft-Chargieröfen verwendet worden. Das neue Ofenkonzept soll die schnelle und verarbeitungsgerechte Erwärmung der Magnesiumrohlinge mit einem um rund $30 \%$ reduzierten Energiebedarf gewährleisten.

Das Konzept: Ein Handlingsystem legt den schmiedefähigen Werkstoff in die Spindelpresse ein. Ein Teil der eingesetzten Umformenergie kann zurückgewonnen und in Kondensatoren gespeist werden. Der Einsatz der für den Schmiedeprozess erforderlichen elektrischen Energie verringert sich dadurch um bis zu $60 \%$. Aus diesen Energieeinsparungen resultiert eine Reduzierung der $\mathrm{CO}_{2}^{-}$ Emissionen von bis zu 111 t/a.
Schmiedeteile aus Magnesium-Knetlegierungen wie $A Z 31$ oder AZ80 sollen eine Gewichtsreduzierung ermöglichen, die im Vergleich zu Stahl bis zu 60 \% und zu Aluminium bis zu einem Drittel beträgt. Gleichzeitig wird gegenüber spanend hergestellten Werkstücken ein sehr gutes Verhältnis zwischen Rohmaterialeinsatz und fertigem Bauteil erzielt, heißt es weiter. Die endkonturnahe Herstellung der geschmiedeten Teile reduziere gleichzeitig den Nachbearbeitungsaufwand, woraus geringere Kosten für Energie und Zerspanwerkzeuge sowie ein verminderter Personalaufwand resultierten. Darüber wiesen Schmiedeteile eine höhere Festigkeit auf, die durch die Verdichtung im Herstellungsprozess und den gerichteten Faserverlauf entlang der Bauteilkontur entstehe. Sie wirke sich insbesondere bei Bauteilen, die einer Biege-Wechsel-Beanspruchung ausgesetzt sind, vorteilhaft aus. Das neue Anlagenkonzept wurde im Rahmen des Umweltinnovationsprogramms mit rund 188.000 Euro gefördert.

OPTIMIERTE DRUCKGUSSLEGIERUNG NACHWUCHSPREIS NEUE WERKSTOFFE FÜR DOMINIK BÖSCH

Dominik Bösch ist mit dem Nachwuchspreis Neue Werkstoffe ausgezeichnet worden. Verliehen wurde der Preis vom Cluster Neue Wertstoffe im Rahmen des 12. Symposiums "Material Innovativ", das am 11. April 2013 in Aschaffenburg stattfand.

Bösch, wissenschaftlicher Mitarbeiter am Lehrstuhl für Allgemeine Werkstoffeigenschaften der Friedrich-Alexander-Universität Erlangen-Nürnberg, hat seine Diplomarbeit bei Audi geschrieben. Er befasste sich in seiner Arbeit mit der Optimierung von Druckgusslegierungen basierend auf dem Legierungssystem Al-Si-Mg. Seine Forschungsergebnisse sollen zeigen, dass bereits eine Zugabe von geringen Mengen an
Kupfer zu einer signifikanten Festigkeitssteigerung führt. Hierdurch sei eine Reduzierung des Bauteilgewichts bis zu $18 \%$ und somit eine deutliche Verbesserung der $\mathrm{CO}_{2}-$ Bilanz möglich. Die aus der Arbeit hervorgegangenen Ergebnisse seien bei Audi in die Entwicklung einer neuen Klasse von hochfesten Aluminiumdruckgusslegierungen für Anwendungen im Automobilbau eingeflossen.

Eine siebenköpfige Jury von Vertretern aus Wirtschaft und Wissenschaft und des Cluster-Managements von Bayern Innovativ bewertete die Arbeiten hinsichtlich ihrer wissenschaftlichen Exzellenz, des industriellen Anwendungsbezugs und der Relevanz zu den thematischen Schwerpunkten im Cluster Neue Werkstoffe. Der diesjährige Preis ist mit 2000 Euro dotiert und wurde von Krauss Maffei Technologies und Oechsler gestiftet. Mit dem Nachwuchspreis wird jedes Jahr eine herausragende Diplom- oder Bachelorarbeit einer Partnerhochschule des Bayerischen Technologieclusters mit Bezug zur industriellen Anwendung auf dem Gebiet der Neuen Werkstoffe prämiert, um den werkstoffwissenschaftlichen Nachwuchs zu fördern sowie innovative und praktikable Ideen einer breiten Interessensgruppe zugänglich zu machen. Die Auszeichnung ist 2013 bereits zum zweiten Mal durch den Cluster Neue Werkstoffe vergeben worden. 